\title{
Effects of Time Dilation on the Measurements of the Hubble Constant
}

\author{
Naser Mostaghel \\ Civil Engineering Department, University of Toledo, Toledo, USA \\ Email: naser.mostaghel@utoledo.edu
}

How to cite this paper: Mostaghel, N. (2018) Effects of Time Dilation on the Measurements of the Hubble Constant. International Journal of Astronomy and Astrophysics, 8, 339-346.

https://doi.org/10.4236/ijaa.2018.84024

Received: October 7, 2018

Accepted: December 7, 2018

Published: December 10, 2018

Copyright $\odot 2018$ by author and Scientific Research Publishing Inc. This work is licensed under the Creative Commons Attribution International License (CC BY 4.0).

http://creativecommons.org/licenses/by/4.0/

\begin{abstract}
We show that, when measuring the Hubble constant by starting the evaluation from the time of the big bang era, the effect of time dilation results in a decrease in the value of the Hubble constant. But when evaluating the Hubble constant by starting the evaluation from the present time, the effect of time dilation results in an increase in the value of the Hubble constant. To elucidate the process, the time dilation is calculated both directly and through Schwarzschild solution of the Einstein equation for the gravitational time dilation. It is concluded that both measured values are valid but because of time dilation, different starting times for the evaluation of the Hubble constant have resulted in different measured values for the Hubble constant.
\end{abstract}

\section{Keywords}

Hubble Constant, Time Dilation, Redshift, Time Symmetry, Gravitation

\section{Introduction}

The recently reported measured value for the Hubble constant by the Plank team [1] is $H_{0}=67.66 \pm 0.42 \mathrm{~km} \cdot \mathrm{s}^{-1} \cdot \mathrm{Mpc}^{-1}$. The Plank team obtains this value for $H_{0}$ by using the cosmic microwave background (CMB) data together with the $\Lambda \mathrm{CDM}$ standard model. Since CMB happens in the recombination era, the process involves evaluating the Hubble time from the surface of last scattering to the present time and then calculating the value for $H_{0}$. In this measurement, the direction of time is future pointing, that is, toward the future as it progresses from the surface of last scattering toward the present time. Thus as the length of time from the center of gravity is increased, the hold of gravity on time is decreased. The evaluated Hubble time intrinsically includes the time dilation.

Another measured value for the Hubble constant, reported by the Reiss team 
[2] is $H_{0}=73.24 \pm 1.7 \mathrm{~km} \cdot \mathrm{s}^{-1} \cdot \mathrm{Mpc}^{-1}$. This team starts its evaluation from the present time by measuring both the redshift and distance first and then calculating the $H_{0}$. In this measurement the redshift increases as one goes back from the present time. Here the direction of time, for evaluation of $H_{0}$, is past pointing, that is, toward the surface of last scattering. Thus as the length of time to the center of gravity is decreased, the hold of gravity on time is increased. In this case the evaluated $H_{0}$ also intrinsically includes the effect of time dilation.

The discrepancy or tension between the above two values of $H_{0}$ has been puzzling [3] [4] [5]. Here we first evaluate a reference Hubble constant without including the time dilation. Then we show that including the time dilation and starting the evaluation from the surface of last scattering proceeding toward the present time yields $H_{0}=67.8236 \mathrm{~km} \cdot \mathrm{s}^{-1} \cdot \mathrm{Mpc}^{-1}$. This value of $H_{0}$ is remarkably consistent with the Plank measured value. Then, still including the time dilation, we reverse the starting point for the evaluation of $H_{0}$. We show that starting the evaluation from the present time proceeding toward the surface of last scattering yields $H_{0}=74.2119 \mathrm{~km} \cdot \mathrm{s}^{-1} \cdot \mathrm{Mpc}^{-1}$ which is also consistent with the value measured by Reiss et al. It is concluded that, because of time dilation, the direction of evaluation of the Hubble time is the cause of discrepancy or tension between the two measured values.

\section{Evaluation of the Reference Hubble Constant}

According to General Relativity (GR) time and space are coupled and a global reversal of coordinate system has no effect on the result. That is, time symmetry is inherent in GR. Because in the above two measurements the reversal of the proper time of a single world-line from a future-pointing to a past-pointing has resulted in different values for the Hubble constant; the time symmetry is broken [6]. As such one cannot use GR in its classical form to address the problem at hand. In the Plank measurement the direction of time is pointing toward the future while in the Reiss measurement the direction of time is pointing toward the past. In the following it is shown that in the Plank measurement, because the time dilation is positive it increases the Hubble time, resulting in a lower value for the Hubble constant. But in the Reiss measurement, because the time dilation is negative, it decreases the Hubble time, resulting in a higher value for the Hubble constant. It should be noted that the Friedmann-Robertson-Walker model (FRW) of the standard model of relativistic cosmology includes both isotropy and homogeneity, Peacock [7]. But the effect of time dilation does not directly show up in the FRW model because the FRW model is based on General Relativity, in which time is symmetric. Below it will be shown that the time dilations for the future pointing and the past pointing measurements have opposite signs. Increasing entropy with the forward flow of time (which is not included in GR) may be the cause of the broken symmetry.

Here we first evaluate a reference Hubble constant not including the effect of time dilation. Then we evaluate the effects of time dilation on the above two measurements. Because of broken time symmetry, as an alternative exploration 
to GR, let $T_{e}(t)$ represent the expanding time part of the expanding universe where $c z$ represents the rate of expansion of the universe. Since the speed of light, $c$, is constant, the redshift, $z$, represents the rate of change of the expanding time, $T_{e}(t)$. It has been shown that the quantity $a_{t}=z^{2} / t$ represents the second rate or the acceleration of $T_{e}(t)$ at the present time [8]. Thus, the spatial velocity, $v(t)$, and the spatial acceleration, $a(t)$, of the expansion of the universe, in this simple model, are given by

$$
\begin{gathered}
v(t)=c z(t) \\
a(t)=c a_{t}(t)=\frac{c(z(t))^{2}}{t}=\frac{(v(t))^{2}}{R(t)}
\end{gathered}
$$

The acceleration, $a(t)$, is a centrifugal acceleration and its effect according to the equivalence principle of GR is equivalent to gravitational acceleration. Thus

$$
a(t)=\frac{(v(t))^{2}}{R(t)}=\frac{c(z(t))^{2}}{t}=\frac{G M}{(R(t))^{2}}=\frac{G M}{c^{2} t^{2}}
$$

Because the sign of $a(t)$ is positive, it represents a repulsive inertia acceleration. The above relation yields

$$
(z(t))^{2}=\frac{G M}{c^{3} t}
$$

Considering the radius of the universe at the time of the big bang to have been equal to $c t_{p}$ where $t_{p}=5.391157549003072 \times 10^{-44} \mathrm{~s}$ represents the Plank time (the smallest unit of time), based on Equation (4) the total mass of our universe, $M$, is given by

$$
M=\frac{c^{3}\left(z_{p}\right)^{2} t_{p}}{G}
$$

where $z_{p}$ represents the Plank redshift. Since the total mass of the universe, $M$, is conserved, Equation (5) implies that

$$
\frac{G M}{c^{3}}=\left(z_{p}\right)^{2} t_{p}=\left(z_{H}\right)^{2} t_{S t}=(z(t))^{2} \frac{t}{2}
$$

where $t_{S t}$ represents the radius of the surface of last scattering (the horizon) and $z_{H}$ represents its corresponding redshift, and $t / 2$ represents the time distance from the big bang at any time $t$.

We evaluate the Plank redshift, $z_{p}$, based on Plank temperature, $\tau_{p}=1.416807993748161 \times 10^{32} \mathrm{~K}$, the black body radiation temperature at the present time, and Wien's displacement law [9] [10] according to the following relations

$$
z_{p}=\frac{\lambda_{o}}{\lambda_{e}}-1=\frac{\tau_{\text {then }}}{\tau_{\text {now }}}-1=\frac{\tau_{p}}{2.72548}-1=\frac{1.41681 \times 10^{32}}{2.72548}=5.19838 \times 10^{31}
$$

From the $\mathrm{CMB}$ radiations at the time of decoupling, the temperature has been estimated to have been about $3000 \mathrm{~K}$, and the black body radiation temperature 
now is $\tau_{\text {now }}=2.72548 \mathrm{~K} \quad[11]$. From relations (7) one obtains

$$
z_{H}=\frac{\tau_{\text {then }}}{\tau_{\text {now }}}-1=\frac{3000}{2.72548}-1=1099.7235
$$

From Equation (6) the radius of the surface of last scattering, $t_{S t}$, is given as

$$
t_{S t}=\frac{\left(z_{p}\right)^{2} t_{p}}{\left(z_{H}\right)^{2}}=1.20462251807 \times 10^{14} \mathrm{~s}
$$

Thus the reference Hubble time, $\left(t_{H}\right)_{r}$, is given by

$$
\left(t_{H}\right)_{r}=t_{0}-t_{S t}=4.35374417748 \times 10^{17} \mathrm{~s}
$$

where $t_{0}=13.8 \mathrm{Gyr}$ represents the assumed age of the universe at the present time. Therefore, the reference Hubble constant, $\left(H_{0}\right)_{r}$, is given by

$$
\left(H_{0}\right)_{r}=\left(\frac{1}{\left(t_{H}\right)_{r}}\right) \frac{\mathrm{Mpc}}{1000}=70.874 \mathrm{~km} \cdot \mathrm{s}^{-1} \cdot \mathrm{Mpc}^{-1}
$$

\section{Evaluation of the Effects of Time Dilation on the Hubble Constant}

Here we show that the effect of time dilation manifests itself in two ways. If we make our measurement toward the present time, the effect of a positive change in time is a negative change in the redshift. But if we make our measurement toward the big bang, the effect of a negative change in time is a positive change in the redshift. Let $\delta t$ represent a small change in time. Since according to Equation (6)

$$
\frac{t}{2}=\frac{\left(z_{p}\right)^{2} t_{p}}{z^{2}}
$$

it follows that

$$
t \pm \delta t=\frac{2\left(z_{p}\right)^{2} t_{p}}{(z \mp \delta z)^{2}}
$$

That is, a positive change in the value of time, $t$, corresponds to a negative change in the value of the redshift, $z$. But a positive change in the value of redshift corresponds to a negative change in the value of time. If we start the measurement from the surface of last scattering to the present time, $t_{0}$, the length of time is $\left(t_{H}\right)_{r}=t_{0}-t_{S t}$. Thus the corresponding change in redshift is given by

$$
\delta z_{1}=z\left(t_{0}\right)-z\left(t_{H}\right)_{r}
$$

Substitutions for $z\left(t_{0}\right)$ and $z\left(t_{H}\right)_{r}$ using Equation (12) yield the change in the redshift as

$$
\delta z_{1}=\sqrt{\frac{2\left(z_{p}\right)^{2} t_{p}}{t_{0}}}-\sqrt{\frac{2\left(z_{p}\right)^{2} t_{p}}{\left(t_{H}\right)_{r}}}=-0.0035782 \mathrm{~s} / \mathrm{s}
$$

Due to initial conditions, $z\left(t_{0}\right)$ is not zero, but its effect cancels out. In 
making the measurement toward the present time one is moving away from the gravitational center in the direction of increasing time, which according to Equation (4) causes a reduction or a negative change in the value of the redshift. Since $\delta z_{1}$ is negative, the change in time according to Equation (13) must be positive. Thus changing the sign of $\delta z_{1}$, one obtains the time dilation as

$$
\Delta t_{1}=2\left(-\delta z_{1} \times 2 \pi t_{0}\right)=1.9581837 \times 10^{16} \mathrm{~s}
$$

Factor 2 in the above equation represents the fact that, from the time of the big bang, the expanding time expands both toward the earth and in the opposite direction. The $2 \pi$ factor represents the fact that, based on Equation (3), the path of expanding time is circular. Now evaluating the Hubble time via evaluation based on measurement from the surface of last scattering toward the present time, including time dilation, one obtains the Hubble time as

$$
\left(t_{H}\right)_{1}=\left(t_{H}\right)_{r}+\Delta t_{1}=4.5495625 \times 10^{17} \mathrm{~s}
$$

where $\left(t_{H}\right)_{r}$ is the reference Hubble time given in Equation (10). Therefore the present time value of the Hubble constant, in this case, is calculated to be

$$
\left(H_{0}\right)_{1}=\frac{1}{\left(t_{H}\right)_{1}} \frac{\mathrm{Mpc}}{1000}=67.824 \mathrm{~km} \cdot \mathrm{s}^{-1} \cdot \mathrm{Mpc}^{-1}
$$

The above value for the Hubble constant is consistent with Plank's value of $H_{0}=67.66 \pm 0.42$.

In the foregoing case the evaluation of the Hubble time, $\left(t_{H}\right)_{1}$, is from the surface of last scattering to the present time, $t_{0}$, on earth. Now we consider evaluation of the Hubble constant through measurement of redshift from the present-time toward the surface of last scattering. Reversing the direction of evaluation of Hubble time causes the change in the redshift to be positive. This is so because in making the measurement going back from the present-time one is moving toward the gravitational center, reducing the length of time and, according to Equation (4), causing an increase or a positive change in the value of the redshift. The length of time to the surface of last scattering is $\left(t_{H}\right)_{r}=t_{0}-t_{S t}$. Thus the change in redshift is

$$
\delta z_{2}=z\left(t_{H}\right)_{r}-z\left(t_{0}\right)=\sqrt{\frac{2\left(z_{p}\right)^{2} t_{p}}{\left(t_{H}\right)_{r}}}-\sqrt{\frac{2\left(z_{p}\right)^{2} t_{p}}{t_{0}}}=0.0035782 \mathrm{~s} / \mathrm{s}
$$

Since $\delta z_{2}$ is positive, the change in time according to Equation (13) must be negative. Thus changing the sign of $\delta z_{2}$ one obtains the time dilation as

$$
\Delta t_{2}=2\left(-\delta z_{2} \times 2 \pi t_{0}\right)=-1.9581837 \times 10^{16} \mathrm{~s}
$$

Consequently the Hubble time including the effect of time dilation is given by

$$
\left(t_{H}\right)_{2}=\left(t_{H}\right)_{r}+\Delta t_{2}=4.1579258 \times 10^{17} \mathrm{~s}
$$

Thus the Hubble constant including the effect of time dilation is given by

$$
\left(H_{0}\right)_{2}=\frac{1}{\left(t_{H}\right)_{2}} \frac{\mathrm{Mpc}}{1000}=74.212 \mathrm{~km} \cdot \mathrm{s}^{-1} \cdot \mathrm{Mpc}^{-1}
$$


The above value for the Hubble constant is consistent with the Reiss, et al. value of $H_{0}=73.24 \pm 1.7$.

\section{Time Dilation Based on the Schwarzschild Solution}

As an approximate check on the numerical values of the time dilations calculated above, assuming the total mass of the universe to be non-rotating and spherically-symmetric, here we explore the use of the Schwarzschild solution of the Einstein equation for the gravitational time dilation between points close and far from the gravitational center. The Schwarzschild solution has been a useful approximation for describing slowly rotating astronomical objects such as stars and planets [12] [13]. The solution is given as

$$
t_{0}=\tilde{t} \sqrt{1-\frac{2 G M}{c^{3} t_{0}}}=\tilde{t} \sqrt{1-\frac{2\left(z_{p}\right)^{2} t_{p}}{t_{0}}}
$$

where $t_{0}$ and $\tilde{t}$ correspond to slow-ticking and fast-ticking clocks respectively. The length of time between the surface of last scattering and the earth is $\left(t_{H}\right)_{r}=t_{0}-t_{S t}$. Therefore, based on Equation (6) the corresponding change in the redshift, $z$, due to time dilation, is given by

$$
\delta \tilde{z}_{1}=\frac{t_{0}}{\tilde{t}_{0}}-\frac{t_{H}}{\left(\tilde{t}_{H}\right)_{r}}=\left|\sqrt{1-\frac{2\left(z_{p}\right)^{2} t_{p}}{t_{0}}}\right|-\left|\sqrt{1-\frac{2\left(z_{p}\right)^{2} t_{p}}{\left(t_{H}\right)_{r}}}\right|=-0.0035808 \mathrm{~s} / \mathrm{s}
$$

where, to avoid imaginary values, as an exploratory evaluation, we have used the absolute values. Thus the time dilation is given by

$$
\Delta \tilde{t}_{1}=2\left(-\delta \tilde{z}_{1} \times 2 \pi t_{0}\right)=1.9596485 \times 10^{16} \mathrm{~s}
$$

Now calculating the Hubble time via evaluation based on measurement from the surface of last scattering toward the present time, including time dilation, one obtains the Hubble time as

$$
\left(\tilde{t}_{H}\right)_{1}=\left(t_{H}\right)_{r}+\Delta \tilde{t}_{1}=4.5497090 \times 10^{17} \mathrm{~s}
$$

where $\left(t_{H}\right)_{r}$ is the reference Hubble time given in Equation (10). Therefore the present time value of the Hubble constant in this case is given by

$$
\left(\tilde{H}_{0}\right)_{1}=\frac{1}{\left(\tilde{t}_{H}\right)_{1}} \frac{\mathrm{Mpc}}{1000}=67.821 \mathrm{~km} \cdot \mathrm{s}^{-1} \cdot \mathrm{Mpc}^{-1}
$$

Reversing the direction of evaluation, the change in redshift due to time dilation is given by

$$
\delta \tilde{z}_{2}=\frac{t_{H}}{\left(\tilde{t}_{H}\right)_{r}}-\frac{t_{0}}{\tilde{t}_{0}}=\left|\sqrt{1-\frac{2\left(z_{p}\right)^{2} t_{p}}{\left(t_{H}\right)_{r}}}\right|-\left|\sqrt{1-\frac{2\left(z_{p}\right)^{2} t_{p}}{t_{0}}}\right|=0.0035808 \mathrm{~s} / \mathrm{s}
$$

Thus the value of time dilation is given by

$$
\Delta \tilde{t}_{2}=2\left(-\delta \tilde{z}_{2} \times 2 \pi t_{0}\right)=-1.9596485 \times 10^{16} \mathrm{~s}
$$


Table 1. Measured and calculated values of Hubble constant.

\begin{tabular}{cccc}
\hline & $\begin{array}{c}H_{0} \\
\mathrm{~km} \cdot \mathrm{s}^{-1} \cdot \mathrm{Mpc}^{-1}\end{array}$ & $\begin{array}{c}\left(H_{0}\right)^{*} \\
\mathrm{~km} \cdot \mathrm{s}^{-1} \cdot \mathrm{Mpc}^{-1} \\
\text { Calculated }\end{array}$ & $\begin{array}{c}\left(\tilde{H}_{0}\right)^{* *} \\
\mathrm{~km} \cdot \mathrm{s}^{-1} \cdot \mathrm{Mpc}^{-1} \\
\text { Calculated }\end{array}$ \\
\hline Plank 2018 & $67.66 \pm 0.42$ & 67.824 & 67.821 \\
Reiss et al. & $73.24 \pm 1.7$ & 74.212 & 74.215 \\
\hline
\end{tabular}

${ }^{*}$ Time dilation is evaluated directly; ${ }^{* *}$ Time dilation is evaluated based on the Schwarzschild solution.

Consequently the Hubble time including the effect of time dilation in this case is given by

$$
\left(\tilde{t}_{H}\right)_{2}=\left(t_{H}\right)_{r}+\Delta \tilde{t}_{2}=4.1577793 \times 10^{17} \mathrm{~s}
$$

Thus the Hubble constant including the effect of time dilation is calculated to be

$$
\left(\tilde{H}_{0}\right)_{2}=\frac{1}{\left(\tilde{t}_{H}\right)_{2}} \frac{\mathrm{Mpc}}{1000}=74.215 \mathrm{~km} \cdot \mathrm{s}^{-1} \cdot \mathrm{Mpc}^{-1}
$$

For comparison, the calculated Hubble constants and the measured ones are presented in Table 1.

\section{Conclusion and Remarks}

Based on the results presented above it is clear that, because of time dilation, the measurement of $H_{0}$ is sensitive to the direction in which the measurement is performed, that is toward the present time or away from it. Therefore, considering the directions of evaluation, both measured values of the Hubble constant are valid. Removal of time dilation would yield a unique value for $H_{0}$. However, because time dilation is inherent in the evaluation of the length of time, its effect cannot be removed through more precise measurements. Also according to Equation (3) the redshift, $z$, that is, the rate of change of the expanding time, $T_{e}(t)$, can represent the repulsive gravity.

\section{Conflicts of Interest}

The author declares no conflicts of interest regarding the publication of this paper.

\section{References}

[1] Aghanim, N., Akrami, Y., Ashdown, M., et al. (2018) Plank 2018 Results. VI. Cosmological Parameters. https://arxiv.org/abs/1807.06209

[2] Riess, A.G., Casertano, S., Yuan, W., et al. (2018) Milky Way Cepheid Standards for Measuring Cosmic Distances and Application to Gaia DR1: Implications for the Hubble Constant. https://arxiv.org/abs/1804.10655

[3] Freedman, W.L. (2017) Cosmology at Crossroads: Tension with the Hubble Constant. https://arxiv.org/abs/1706.02739

[4] Donna Weaver, D. and Villard, R. (2018) Improved Hubble Yardstick Gives Fresh Evidence for New Physics in the Universe. NASA. 
https://www.nasa.gov/feature/goddard/2018/improved-hubble-yardstick-gives-fresh -evidence-for-new-physics-in-the-universe/

[5] Devlin, H. (2018) The Answer to Life, the Universe and Everything Might Be 73. Or 67. The Guardian.

https://www.theguardian.com/science/2018/may/10/the-answer-to-life-the-universe -and-everything-might-be-73-or-67

[6] Ripalda, J.M. (2010) Time Reversal and Negative Energies in General Relativity. arXiv:gr-qc/9906012, v14.

[7] Peacock, J. (1999) Cosmological Physics. Cambridge University Press, Cambridge.

[8] Mostaghel, N. (2018) The Role of Time in Cosmic Expansion. American Journal of Astronomy and Astrophysics, 6, 9-21.

[9] NIST, CODATA VALUE: Wien Wavelength Displacement Law Constant. http://physics.nist.gov/cgi-bin/cuu/Value?bwien

[10] Wikipedia, Wien's Displacement Law. https://en.wikipedia.org/wiki/Wien\%27s_displacement_law

[11] Fixsen, D.J. (2009) The Temperature of the Cosmic Microwave Background. The Astrophysical Journal, 707, 916-920. https://doi.org/10.1088/0004-637X/707/2/916

[12] Wikipedia, Gravitational Time Dilation. https://en.wikipedia.org/wiki/Gravitational_time_dilation

[13] Wikipedia, Schwarzschild Solution. https://en.wikipedia.org/wiki/Schwarzschild_metric 